\title{
RASSEGNA
}

\section{Intraductal papillary mucinous neoplasm of the pancreas. Personal series and synthetic review ${ }^{\text {is }}$}

\section{Neoplasia mucinosa papillare intraduttale del pancreas. Casistica personale e revisione sintetica}

\author{
F. Gallucci ${ }^{a}$, D. Avolio ${ }^{a}$, R. de Ritis ${ }^{b}$, L. Ferrara $^{a}$, U. Valentino ${ }^{a}$, G. Uomo ${ }^{a, *}$
}

a Department of Internal Medicine and Radiology (Unit 3), Cardarelli Hospital, Napoli, Italy

${ }^{\mathrm{b}}$ Department of Internal Medicine and Radiology, Cardarelli Hospital, Napoli, Italy

Received 30 November 2011; accepted 23 February 2012

Available online 20 March 2012

\section{KEYWORDS \\ Pancreatic neoplasm; Mucinous pancreatic \\ tumor; \\ Pancreatic cyst; \\ Diagnosis; \\ Treatment.}

\begin{abstract}
Summary Intraductal papillary mucinous neoplasms (IPMNs) are rare pancreatic tumors, accounting for less than $1-2 \%$ of all neoplasms of the pancreas. The main characteristic of IPMNs is their favorable prognosis, as these pre-malignant or malignant lesions are usually slow-growing tumors and radical surgery is frequently possible. According to the localization of the lesions, three different tumor types have been identified: the main-duct IPMN, the branch-duct IPMN and the mixed-type IPMN (involving both the main pancreatic duct and the side branches). IMPNs do not present pathognomonic signs or symptoms. The obstruction of the main pancreatic duct system may cause abdominal pain and acute pancreatitis (single or recurrent episodes). The tumor may be incidentally discovered in asymptomatic patients, particularly in those with branch-duct IPMNs. In clinical practice, any non-inflammatory cystic lesion of the pancreas should be considered as possible IPMN. Computed tomography, magnetic resonance imaging with cholangiopancreatography and endoscopic ultrasonography can localize an IPMN and assess its morphology and size. The choice between non-operative and surgical management depends on the risk of malignancy and on the definitive distinction between benign and malignant IPMNs. Main-duct IPMNs have a high risk of malignant degeneration, especially in older patients. The clinical and radiological features, as well as treatment and outcome, of eight patients with IPMN (five with main-duct, two with branch-duct and one with mixed-type) observed by the authors over the last ten years are presented.
\end{abstract}

(c) 2012 Elsevier Srl. All rights reserved.

\footnotetext{
it Poster award at the XVI National Congress of FADOI, Firenze 2011, May, 15-18.

* Corresponding author: Internal Medicine Department, Unit 3, Cardarelli Hospital, Via Cardarelli 9 - 80131 Napoli, Italy.

E-mail: g.uomo@aocardarelli.it (G. Uomo).
} 


\section{Introduction}

Intraductal papillary mucinous neoplasms (IPMNs) are rare pancreatic tumors, accounting for less than $1-2 \%$ of all neoplasms of the pancreas [1,2]. Among the uncommon exocrine tumors of the pancreas, IPMNs have received increasing attention in recent years because of their favorable prognosis, obscure nature and their relationship with pancreatic adenocarcinoma. In addition, over the last ten years the number of diagnosed IPMNs has significantly increased and this tumor is more commonly recognized, even in asymptomatic patients, as an incidental finding. IMPNs now represent $10-30 \%$ of all resectable pancreatic tumors in high-volume referral surgical centers [3]. Physicians should become familiar with IPMNs, as it is possible that affected patients are admitted into internal medicine wards because of non-specific, general complaints or because of an incidentally discovered "cystic lesion" of the pancreas. This article presents an overview of the pathological/clinical features and diagnostic/therapeutic problems of IMPNs with a series of eight patients.

\section{Pathology}

IPMNs of the pancreas are characterized by the intraductal proliferation of neoplastic mucinous cells, which usually form papillae, and the cystic dilation of the pancreatic ducts, thus forming a clinically and macroscopically detectable mass [4]. IPMNs are grossly visible $(\geq 1 \mathrm{~cm})$, mucin-producing neoplasms that arise in the main pancreatic duct and/or its branches. Two different tumor types were initially identified: the "mainduct" IPMN and the "branch-duct" IPMN [1,2]. Main-duct IPMNs may be associated with contemporary involvement of the side branches ducts; as a consequence, a third anatomoclinical subtype of IMPN, termed "mixed" type, has been identified [5]. Macroscopically, the main-duct IPMN usually presents as a dilated, mucin-filled main pancreatic duct or as a cystic-like lesion along the main pancreatic duct [6]. The usual location is in the proximal portion of the pancreas, even if the tumor has spread to the entire main pancreatic duct. Sometimes the mucus may extrude through a bulging ampulla, and it is clearly identified at duodenoscopy (Fig. 1). The branch-duct IPMN frequently involves the side branches of the uncinate

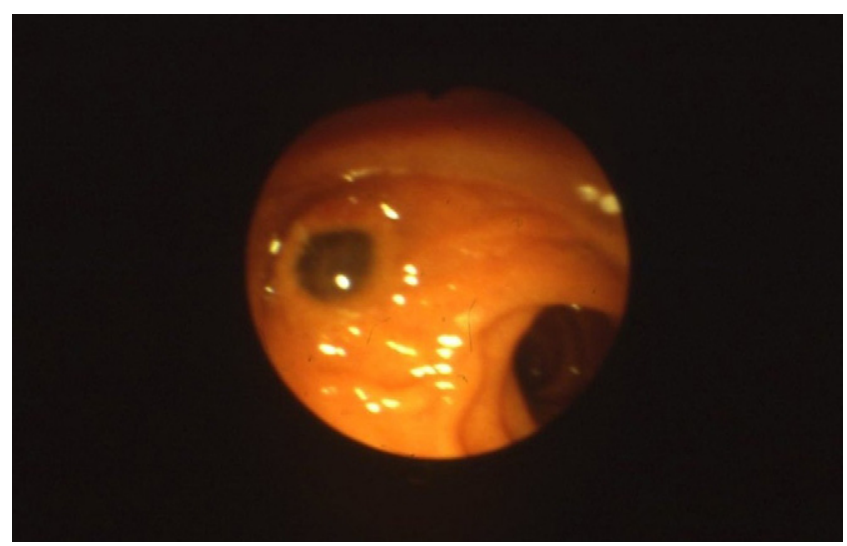

Figure 1 Duodenoscopy: characteristic mucin extrusion through the ampulla of Vater. process, but it can also be observed in the remaining portions of the gland, with the possibility of the multifocal involvement of two or more distant side branches. Branch-duct IMPNs appear as cystic lesions communicating with a non-dilated main pancreatic duct.

According to the "Morphology Code of the International Classification of Diseases for Oncology (ICD-O) and the Systematic Nomenclature of Medicine" (http://snomed.org), there are different pathologic forms:

a) Intraductal papillary-mucinous adenoma $(845 / 0)$

b) Intraductal papillary-mucinous neoplasm with moderate dysplasia (845/1)

c) Intraductal papillary-mucinous carcinoma (845/3) with two subtypes: - non-invasive (845/2) and - invasive $(845 / 3)$

Tumor behavior is coded as follows: 0 for benign tumors; 1 for unspecified, borderline or uncertain behavior; 2 for in situ carcinoma; 3 for malignant tumors. To simplify, IPMNs may be divided into benign (adenoma and borderline) and malignant (in situ carcinoma and invasive carcinoma) $[7,8]$. The latter type (invasive carcinoma) accounts for more than $40 \%$ of the resected specimens at a rate of $43 \%$ for main-duct IPMN and $15 \%$ for branch-duct type [6]. Recently, the International Consensus Guidelines on IPMNs [5] stated that main-duct type and branch-duct type are associated with malignancy in $70 \%$ and $25 \%$ of the cases, respectively. Thus, side-branch IPMNs have a low risk for malignant transformation. Histologic sub-classification based upon the morphology of the proliferative nodules and papillae identifies five types of IPMNs: gastric foveolar type, intestinal type (Fig. 2), pancreatobiliary type, intraductal oncocytic papillary neoplasm, and intraductal tubulopapillary neoplasm $[9,10]$. It is not uncommon to discover multiple histologic types with multiple grades of dysplasia within the same surgical specimen [7].

\section{Molecular Biology}

Over the last ten years many studies have shown genetic alterations in ductal pancreatic adenocarcinoma [11]. Some of these alterations have also been described in IPMNs. The mutational activation of the K-ras oncogene was observed in 15 out of $23(65 \%)$ resected IMPNs, while the loss of

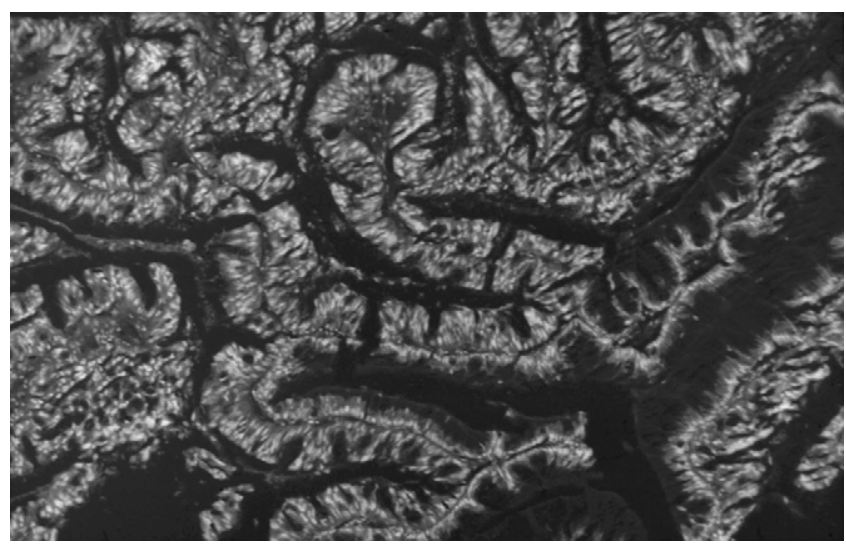

Figure 2 Intestinal-type IPMN resembling villous neoplasms showing columnar epithelial cells with severe epithelial dysplasia (H\&E staining). 
heterozygosity of the $\mathrm{p} 16$ oncosuppressor gene was found in $12.5 \%$ of adenoma and $75 \%$ of carcinoma IMPNs, respectively [12]. Similar results were observed by Yang and co-workers [13]: K-ras mutations were examined in 41 patients (resected specimens) and were detected in $20.8 \%$ of benign IPMNs and in $61.5 \%$ of malignant IPMNs. Another study [14] showed that K-ras and p53 mutations were present in $71 \%$ and $6 \%$ of 52 operated IPMNs, respectively. In vivo studies show slightly different results. Mizuno and co-workers [15] evaluated K-ras mutations in the pancreatic juice in a group of 53 IMPNs, and they observed mutations in $80 \%$ of the carcinoma group and $71 \%$ of the adenoma group. IPMN-cells secrete a thick mucin that causes dilatation of the pancreatic ducts. Recent studies identified different expression of various mucins in IPMNs with different degrees of malignancy. Mucin-1, mucin-4 and mucin-5AC were more frequently associated with invasive carcinoma, and mucin-2 was found only in the intestinal type and more frequently in adenomas or borderline IPMNs $[13,16-18]$. The composition of mucin products may be investigated in surgical specimens and in vivo by means of endoscopic ultrasound-guided fine-needle aspiration [19]. This is critically important as it is possible to identify a "molecular" prognosis to plan the timing and strategy of surgical treatment (limited pancreatectomy, extended resection, lymphoadenectomy).

\section{Clinical features}

IMPNs do not present pathognomonic signs or symptoms. The obstruction of the main pancreatic duct system may cause abdominal pain, pancreatitis, steathorrea, jaundice, diabetes and weight loss $[2,3,5,7]$. The last three symptoms have been described more frequently in patients with malignant IPMNs [3,20]. Asymptomatic patients account for $13.5 \%$ [21] of invasive main-duct IPMNs. Patients affected by branch-duct IPMN are often completely asymptomatic, and the lesion is incidentally discovered during ultrasound or during other radiologic examinations that are performed for various reasons [22,23]. A recent report on a large series of branch-duct IMPNs [23] showed that the neoplasm was incidentally discovered in $40 \%$ of the patients and that jaundice is more frequent in patients with invasive tumors (12.5\% vs. $1.8 \%$ in benign lesions). Abdominal pain is predominant in patients with benign tumors ( $45 \%$ vs. $25 \%$ in malignancy). In clinical practice, it is not uncommon to observe patients with a "cystic lesion" of the pancreas with non-specific symptoms. First, it is necessary to exclude pseudocysts (lesions not presenting on the lining of the wall) such as those related with acute and chronic pancreatitis, paraduodenal wall cysts and infection-related pseudocysts. Then, the differential diagnosis of "true" cysts of the pancreas (lined by epithelium, acinar cells and other cells) includes several pathologic features (Table 1).

\section{Imaging}

Diagnostic procedures for IPMN include endoscopic retrograde cholangiopancreatography (ERCP), computed tomography $(C T)$, magnetic resonance imaging (MRI) with cholangiopancreatography (MRCP) and endoscopic ultrasonography (EUS). In the recent past, ERCP represented the gold
Table 1 Types of "true" (non-inflammatory) cystic lesions of the pancreas; these cysts may be lined by epithelium, acinar cells or other cells.

a) Cysts with mucinous epithelium

Intraductal papillary mucinous neoplasms with its variants Mucinous cystic neoplasms

Mucinous non-neoplastic cysts (mucoceles and retention cysts)

Serous (clear-cell) cystic tumors

Serous cystadenoma and cystadenocarcinomas

Von Hippel Lindau-associated pancreatic cysts

Squamous-lined cysts

Lymphoepithelial cysts

Epidermoid and dermoid cysts

Squamoid cyst of pancreatic ducts

b) Cysts lined by acinar cells

Acinar cell cystadenoma and cystadenocarcinomas

Endothelial-lined cysts

Lymphangiomas

Degenerative or necrotic changes in solid tumors

Solid-pseudopapillary tumor

Cystic change in ordinary ductal adenocarcinoma

Cystic pancreatic endocrine neoplasia (islet cell tumors)

Cystic change in other invasive carcinomas and cystic mesenchymal tumors

c) Other rare cystic lesions

Cystic hamartomas

Enterogenous (congenital; duplication) cysts and duodenal diverticula

Endometriotic cyst

Secondary tumors

Congenital or developmental cysts

Unclassified cysts

standard, at least for the main-duct neoplasms. The detection of bulging ampulla of Vater, mucin secretion, and a dilated main pancreatic duct can lead physicians to a direct diagnosis. The relative invasiveness and poor visualization of the entire main pancreatic duct and side branches have relegated this procedure to an optional examination. Currently, the vast majority of IPMNs are characterized by means of cross-sectional imaging studies [9,22,24,25]. CT and MRI/ MRCP can localize the tumor and assess its morphology and size. The characteristic feature of IPMNs is cystic dilation of the main pancreatic duct and/or of the side branch ducts (Figs. 3-5). Filling defects representing nodules and papillary projections may be found within the cystic lesions (Fig. 6). In addition, CT and MRI with contrast enhancement successfully assess the relationship of IPMN with local vessels and adjacent organs. MRCP is particularly useful in the characterization of single or multifocal branch-duct neoplasms. This procedure is able to examine the communication between the main pancreatic duct and the cystic lesions. The quality of the MRCP images is improved by the use of secretin stimulation. EUS may be very useful in patients who do not have a definite diagnosis after CT/MRCP. EUS can assess the main pancreatic duct and the presence of nodules and papillae in the main duct with limits only in patients with previous gastrointestinal surgery. In addition, EUS may be 


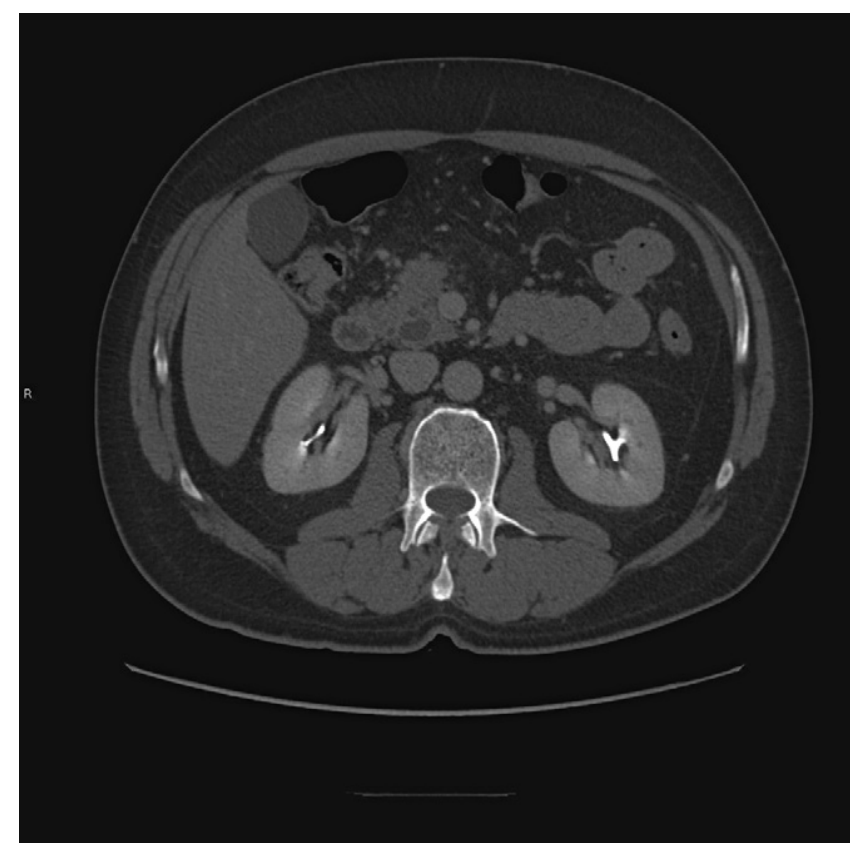

Figure 3 Contrast-enhanced CT scan; main-duct IPMN: the presence of multiple cystic lesions at the head of the pancreas.

completed with a fine-needle aspiration and cytologic and molecular/biochemical exams on the mucin/solid tissue samples [5,19]. International consensus guidelines [5] suggested that EUS should be considered as a second-level

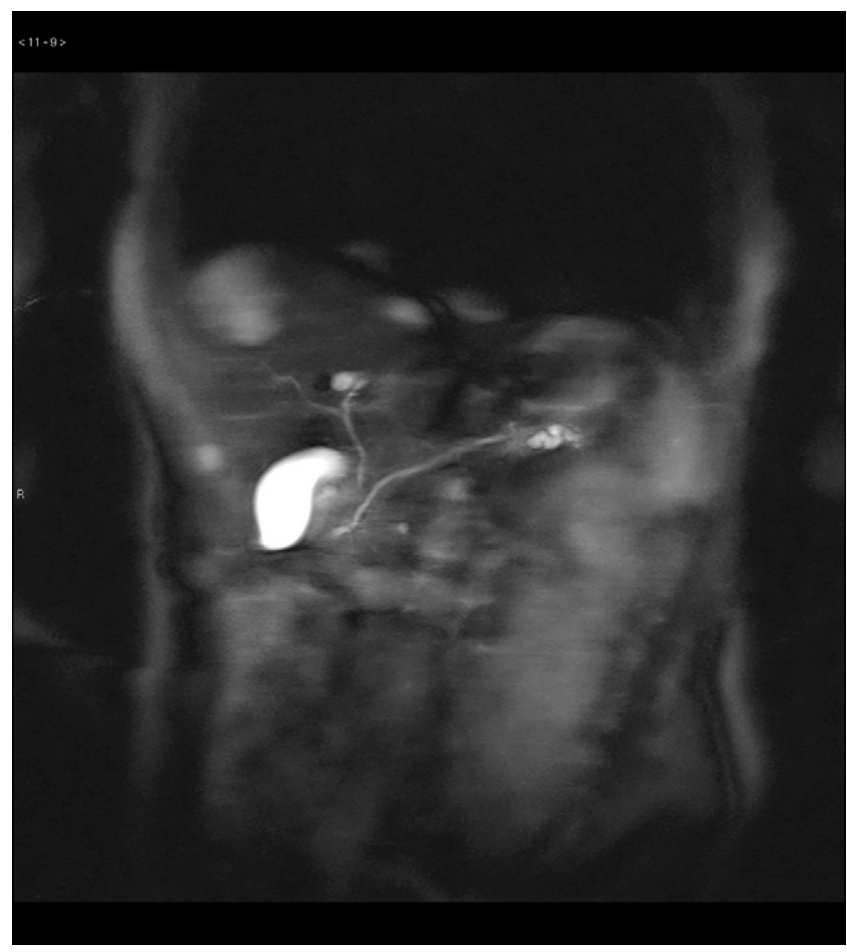

Figure 4 MRCP; IPMN of the distal main pancreatic duct (tail of the pancreas).

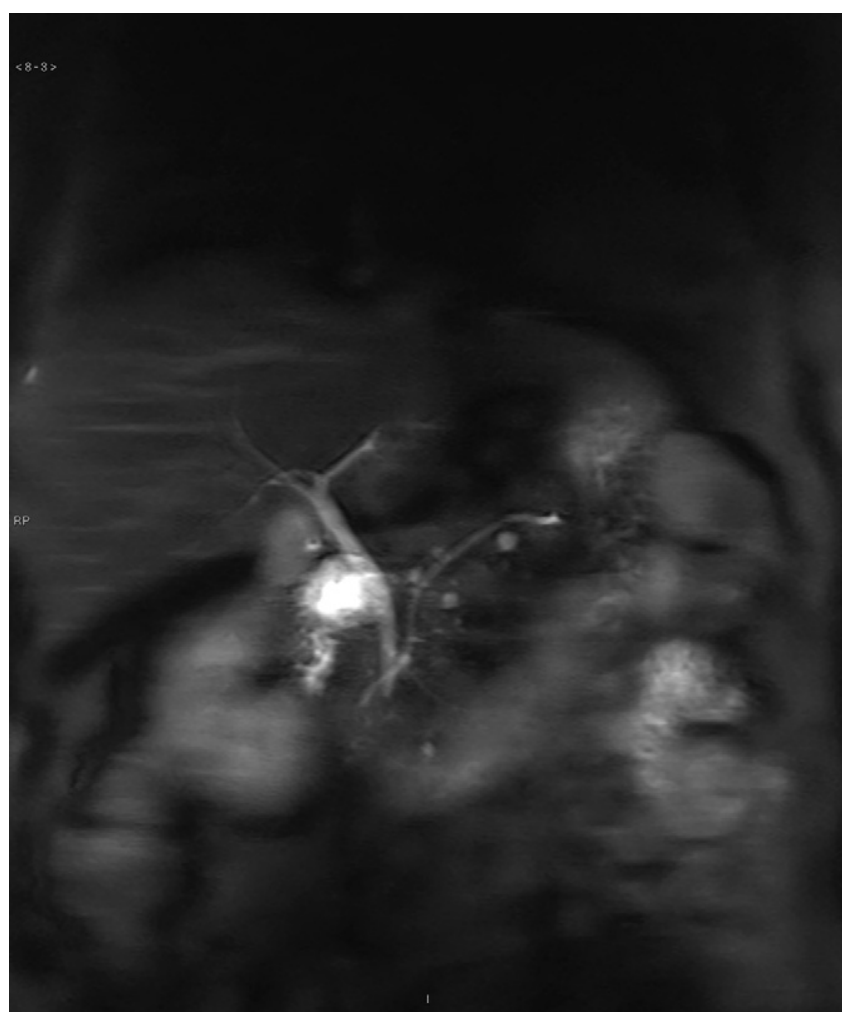

Figure $5 \quad M R C P$ with secretin stimulation $(1 \mathrm{lU} / \mathrm{Kg} / \mathrm{body}$ weight); IPMN of the side branches (body-tail of the gland) in pancreas divisum.

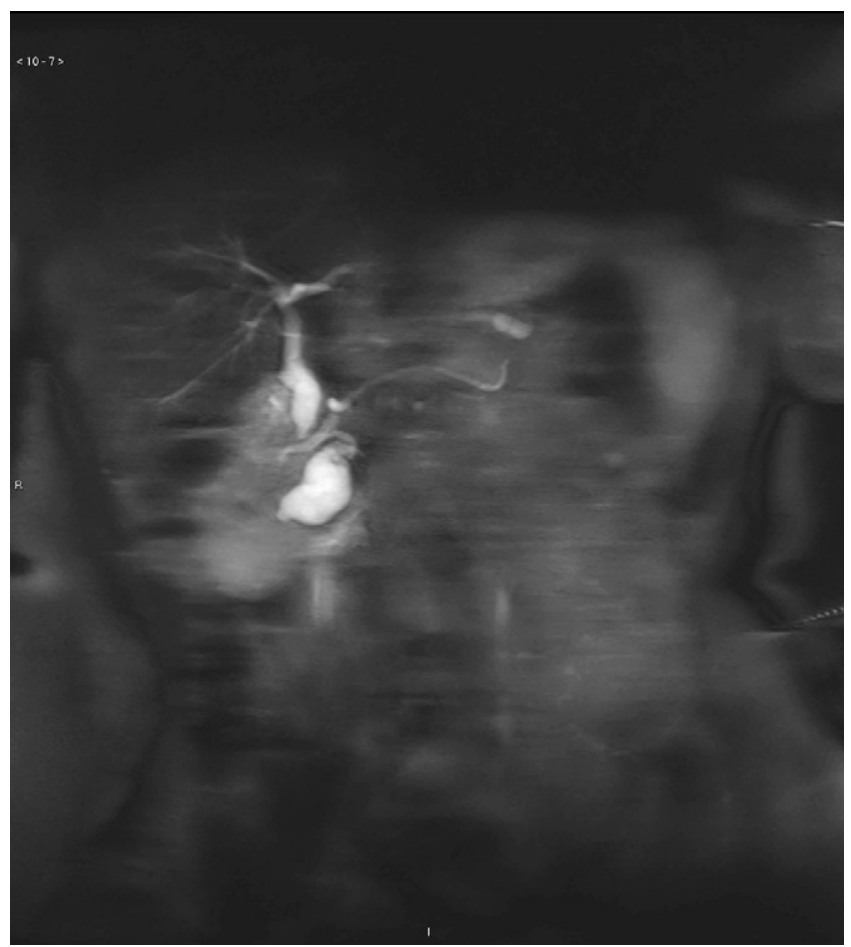

Figure $6 \quad M R C P$ with secretin stimulation $(1 \mathrm{lU} / \mathrm{Kg} /$ body weight); IPMN of the uncinate process: the presence of filling defects within the cystic lesion representing nodules and papillary projections. 
procedure, which should be performed only in selected cases. Alternatively, over the last few years, EUS with fine-needle aspiration is more often utilized as valuable complementary pre-operative procedure in patients with an already established diagnosis. In recent years, contrastenhanced abdominal ultrasound, intraductal pancreatic endoscopy and intraductal endosonography have been introduced for diagnostic purposes, but experience with these procedures is still limited and additional studies are needed [26].

\section{Treatment}

The choice between non-operative and surgical management strictly depends on the risk of malignancy and on the definitive distinction between benign and malignant IPMNs. Despite the fact that our understanding of the natural history of IPMNs is incomplete, recent data indicate that main-duct and branch-duct IPMNs show significant differences in terms of biological behavior, with main-duct IPMNs having a high risk of malignant degeneration. The frequency of malignancy (in situ and invasive) in main-duct IPMNs ranges between 60 and $92 \%$, with a mean of $70 \%$, and approximately two-thirds of these malignant neoplasms have been invasive [5]. Malignancy is more common in older patients, in symptomatic patients (mainly those presenting with jaundice and/or worsening of diabetes), in neoplasms with higher dilation of the main pancreatic duct, or with the presence of mural nodules and eggshell calcifications [7,18,20]. Evidence of 'clonal progression' in these neoplasms [12] and the age difference between patients with malignant and benign lesions [10] are indicative that most, if not all, benign main-duct IPMNs may progress into invasive cancer. Furthermore, the long-term follow-up of resected patients shows excellent survival for benign and non-invasive neoplasms and a 5-year survival between 36 and $60 \%$ for invasive carcinomas $[7,27,28]$. Based upon these considerations, there are no concerns about the management of main-duct neoplasms, while the management of branch-duct tumors is still uncertain. International guidelines stated that all suspected type-1 (main-duct) and type-3 (mixed-type) IPMNs should be resected, even in asymptomatic patients [5]. Various surgical procedures, such as pancreaticoduodenectomy, left pancreatectomy or total pancreatectomy (according to the site and extent of the neoplasm) with lymph node dissection, must be performed
$[3,6,7,21,29]$. An intraoperative frozen section assessment of the pancreatic resection margin can guide the extent of the resection, keeping in mind that IPMNs are often multifocal. For these reasons, oncological radicality is of paramount importance and limited resections and/or laparoscopic approaches are occasionally indicated in this setting $[21,28,29]$. Branch-duct IPMNs have a less harmful biological behavior with the frequency of malignancy ranging from $6 \%$ to $46 \%$, (mean $25 \%$ ) and of invasive cancer from $0 \%$ to $31 \%$ (mean15\%) [5,18]. This suggests a less aggressive surgical approach and the possibility of conservative management and careful follow-up [23]. Some malignancy-related parameters have been proposed: the presence of symptoms (in particular recent-onset or worsened diabetes), lesion lesion size $>3.5 \mathrm{~cm}$, the presence of nodules or thick walls, and a carbohydrate antigen 19.9 serum level $>25 \mathrm{U} / \mathrm{l}$. In a recent prospective study [30] in which these parameters were utilized, surgery was indicated in $18.3 \%$ of 109 patients with branch-duct IPMNs; in the remaining group (89 cases, $81.7 \%$ ), only 5 patients (5.6\%), after a mean follow up of 18.2 months, showed an increase in lesion size and underwent surgery. Similar results were observed in another recent study involving 194 patients from South Korea [31]: 34 patients (17.5\%) were immediately operated on and 18 (11.8\%) underwent surgical resection after a median follow up of 12.7 months. After surgery on non-invasive IPMNs (branch- and main-duct varieties), recurrence is rare $(<6 \%)$, while recurrence occurs in 50-65\% of patients resected for invasive IPMNs [20,22]. The International Association of Pancreatology guidelines [5] for the management of pancreatic IPMNs suggested a yearly follow-up for brunch-duct neoplasms if the lesion is < $10 \mathrm{~mm}$ in size, a 6-12 monthly follow-up for lesions between 10 and $20 \mathrm{~mm}$, and a 3-6 monthly follow-up for lesions $>20 \mathrm{~mm}$. The suggested diagnostic procedures for lesions up to $10 \mathrm{~mm}$ in size are CT/MRCP, while for lesions > $10 \mathrm{~mm}$, EUS with fine-needle aspiration cytology is added to the protocol. Additionally, branch-duct IPMNs are frequently multifocal, and as a consequence, when surgery is planned, total pancreatectomy or subtotal extensive pancreatectomy is required to perform radical surgery. Therefore, it remains questionable whether these high-risk surgical procedures, which are associated with secondary definite exocrine and endocrine insufficiency, are appropriate in elderly and asymptomatic patients suffering with pancreatic tumors with uncertain biological behavior [32].

Table 2 Main clinical characteristics of patients; AP: acute pancreatitis.

\begin{tabular}{lccccccc}
\hline Patients & Age & Gender & IPMN type & Related symptoms & Comorbidity & Treatment & Follow-up \\
\hline 1 & 79 & M & main-duct & AP (single episode) & pulmonary disease & conservative & died 6 yrs later \\
\hline 2 & 63 & F & main-duct & AP (single episode) & diabetes & surgery & alive at 2,4 yrs \\
\hline 3 & 72 & F & mixed & recurrent AP & coronary disease & surgery & alive at 3,1 yrs \\
\hline 4 & 59 & F & main-duct & abdominal pain & diabetes & surgery & alive at 3,7 yrs \\
\hline 5 & 63 & F & main-duct & recurrent AP & no & surgery & alive at 2,7 yrs \\
\hline 6 & 62 & F & branch-duct & AP (single episode) & liver cirrhosis (transplanted) & conservative & died 2,6 yrs later \\
\hline 7 & 60 & M & main-duct & no symptoms & peptic ulcer & surgery & alive at 3,5 yrs \\
\hline 8 & 54 & M & branch-duct & no symptoms & no & conservative & alive at 2,4 yrs \\
\hline
\end{tabular}




\section{Personal series}

Our series included eight patients observed over the last ten years. The main clinical characteristics are shown in Table 2. The mean age was 64 years (range 54-79). The clinical presentation was related to acute pancreatitis in five patients: three with a single episode and two with recurrent attacks; one additional patient was observed because of nonspecific dull abdominal pain. Only two patients (one with main-duct and one with branch-duct IPMN) were asymptomatic. A complete laboratory work-up, including serum tumor markers, was performed in all cases. The first two patients in this series underwent ERCP. All of the patients were examined by means of abdominal ultrasonography, contrast-enhanced CT scan and MRCP (five patients had secretin stimulation). Three patients were also examined with EUS and fine-needle aspiration. Five-year and global mortality are $12.5 \%$ and $25 \%$, respectively. Both patients who died during the follow-up refused surgery. For an additional asymptomatic patient with an incidentally discovered branch-duct IPMN $(2 \mathrm{~cm}$ at the tail of the gland) surgical resection was not indicated; the size of this lesion remained stable during the follow-up (last MRCP performed two years after the diagnosis). Thus, surgical resection was performed in 5 out of 8 patients (62.5\%). The post-operative course was complicated by pleural effusion and pneumonitis in one patient, by pancreatic fistula in two patients (recovery in two and three months, respectively). No recurrence has been registered in the six patients remaining.

\section{Conclusions}

IPMN should be considered in any non-inflammatory cystic lesion of the pancreas and in patients suffering from a single episode or recurrent episodes of acute pancreatitis without a definite etiological factor. Physicians should become familiar with IPMNs, as these pre-malignant or malignant lesions are usually slow-growing tumors. As a consequence, curative resection is possible for the majority of cases, both for the more aggressive type (main-duct IPMN) and in the more favorable tumors (branch-duct type). Because malignancy is more common in older patients, the chances of complete resection often depend on comorbidity and on the anesthesiological class-risk more than local invasiveness.

\section{References}

[1] Kloppel G, Solcia E, Longnecker DS, Capella C, Sobin LH. Histological Typing of Tumours of the Exocrine Pancreas. In: World Health Organization International Histological Classification of Tumors. $2^{\text {nd }}$ ed., Berlin: Springer; 1996. p. 11-20.

[2] Hruban RH, Takaori K, Klimstra DS, Adsay NU, Albores-Saavedra J, Blankin AV, et al. An illustrated consensus on the classification of pancreatic intraepithelial neoplasia and intraductal papillary mucinous neoplasms. Am J Surg Pathol 2004;28:977-87.

[3] Crippa S, Salvia R, Partelli S, Armatura G, Bassi C. Intraductal papillary mucinous neoplasms of the pancreas. In: Shrikhande SV, Friess H, Buchler MW, editors. Surgery of Pancreatic Tumors. New Delhi: BI Publications; 2008. p. 289-98.

[4] Yamaguchi H, Inoue T, Eguchi T, Miyasaka Y, Ohuchida K, Mizumoto $\mathrm{K}$, et al. Fascin overexpression in intraductal papillary mucinous neoplasms (adenomas, borderline neoplasms, and carcinomas) of the pancreas, correlated with increased histological grade. Modern Pathology 2007;20:552-61.

[5] Tanaka M, Chari S, Adsay V, Fernandez-del Castillo C, Falconi M, Shimizu M, et al. International consensus guidelines for management of intraductal papillary mucinous neoplasms and mucinous cystic neoplasms of the pancreas. Pancreatology 2006;6:17-32.

[6] Shi C, Hruban RH. Intraductal papillary mucinous neoplasm. Hum Pathol 2011 July 19 [Epub ahed of print; PMID 21777948].

[7] Salvia R, Fernandez-del Castillo C, Bassi C, Thayer SP, Falconi M, Mantovani $\mathrm{W}$, et al. Main-duct intraductal papillary mucinous neoplasms of the pancreas: clinical predictors of malignancy and long-term survival following resection. Ann Surg 2004; 239:678-85.

[8] Weitz J, Buchler MW. Branch intraductal papillary mucinous neoplasms: just the tip of the iceberg. Gut 2008;57:1490-1.

[9] Katabi N, Klinstra DS. Intraductal papillary mucinous neoplasms of the pancreas: clinical, pathological features and diagnostic approach. J Clin Pathol 2008;61:1303-13.

[10] Adsay NV. Cystic lesions of the pancreas. Modern Pathology 2007;20:S71-93.

[11] Vitone IJ, Greehalf W, McFaul CD, Ghaneh P, Neoptolemos JP. The inherited genetics of pancreatic cancer and prospects for secondary screening. Best Pract Res Clin Gastroenterol 2006; 20:253-83.

[12] Wada K, Takada T, Yasuda H, Amano H, Yoshida M, Sugimoto M, et al. Does "clonal progression" relate to the development of intraductal papillary mucinous tumours of the pancreas? J Gastrointest Surg 2004;8:289-96.

[13] Jang JY, Park YC, Song YS, Lee SE, Hwang DW, Lim CS, et al. Increased K-ras mutation and expression of S100A4 and MUC2 protein in the malignant intraductal papillary mucinous tumor of the pancreas. Hepatobiliary Pancreat Surg 2009;16: 668-74.

[14] Chadwick B, Willmore-Payne C, Tripp S, Layfield LJ, Hirschowitz $\mathrm{S}$, Holden J. Histologic, immunohistochemical, and molecular classification of 52 IPMNs of the pancreas. Appl Immunohistochem Mol Morphol 2009;17:31-9.

[15] Mizuno O, Kawamoto H, Yamamoto N, Horiguchi S, Tsutsumi K, Fujii $\mathrm{M}$, et al. Single-pattern convergence of $\mathrm{K}$-ras mutation correlates with surgical indication of intraductal papillary mucinous neoplasms. Pancreas 2010;39:617-21.

[16] Kanno A, Satoh K, Kimura K, Hirota M, Umino J, Masamune A, et al. The expression of MUC4 and MUC5AC is related to the biologic malignancy of intraductal papillary mucinous neoplasms of the pancreas. Pancreas 2006;33:391-6.

[17] Kobayashi N, Inamori M, Fujita K, Fujisawa T, Fujisawa N, Takahashi $\mathrm{H}$, et al. Characterization of $\mathrm{K}$-ras gene mutations in association with mucinous hypersecretion in intraductal papillary-mucinous neoplasms. J Hepatobiliary Pancreat Surg 2008;15:169-77.

[18] Lee JH, Lee KT, Park J, Bae SY, Lee KH, Lee JK, et al. Predictive factors associated with malignancy of intraductal papillary mucinous pancreatic neoplasms. World J Gastroenterol 2010; 16:5353-8.

[19] Carrara S, Cangi MG, Arcidiacono PG, Perri F, Petrone MC, Mezzi G, et al. Mucin expression pattern in pancreatic diseases: findings from EUS-guided fine-needle aspiration biopsies. Am J Gastroenterol 2011;106:1359-63.

[20] Bassi C, Sarr MG, Lillemoe KD, Reber HA. Natural history of intraductal papillary mucinous neoplasms (IPMN): current evidence and implications for management. J Gastrointest Surg 2008;12:645-50.

[21] Partelli S, Fernandez-del Castillo C, Bassi, Mantovani W, Thayer SP, Crippa S, et al. Invasive Intraductal papillary mucinous carcinomas of the pancreas: predictors of survival and the role of lymph node ratio. Ann Surg 2010;251:477-82.

[22] Salvia R, Crippa S, Partelli S, Armatura G, Malleo G, Paini M, et al. Differences between main-duct and branch-duct intraductal 
papillary mucinous neoplasms of the pancreas. World J Gastrointest Surg 2010;2:342-6.

[23] Rodriguez JR, Salvia R, Crippa S, Warshaw AL, Bassi C, Falconi M, et al. Brach-duct intraductal papillary mucinous neoplasms: observations in 145 patients who underwent resection. Gastroenterology 2007;133:72-9.

[24] Manfredi R, Grazioli R, Motton M, Mantovani W, Baltieri S, Tognolini A, et al. Main pancreatic duct intraductal papillary mucinous neoplasms: accuracy of MR imaging in differentiation between benign and malignant tumours compared with histopathologic analysis. Radiology 2009;253:106-15.

[25] Tirkes T, Akisik F, Tann M, Balci NC. Imaging of the pancreas with secretin-enhancement. Top Magn Reson Imaging 2009;20:19-24.

[26] Yamao K, Ohashi K, Nakamura T, Suzuki T, Sawaki A, Hara K, et al. Efficacy of peroral pan- creatoscopy in the diagnosis of pancreatic diseases. Gastrointest Endosc 2003;57:205-9.

[27] Chari S, Yadav D, Smyrk TC, DiMagno EP, Miller LJ, Massimo M, et al. Study of recurrence after surgical resection of intraductal papillary mucinous neoplasm of the pancreas. Gastroenterology 2002;123:1500-7.

[28] Sohn TA, Yeo CJ, Cameron JL, Hruban RH, Fukushima N, Campbell KA, et al. Intraductal papillary mucinous neoplasms of the pancreas: an updated experience. Ann Surg 2004;239: 788-99.

[29] Crippa S, Partelli S, Falconi M. Extent of surgical resections for intraductal papillary mucinous neoplasms. World J Gastrointest Surg 2010;2:347-51.

[30] Salvia R, Crippa S, Falconi M, Bassi C, Guarise A, Scarpa A, et al. Branch-duct intraductal papillary mucinous neoplasm of the pancreas: to operate or not to operate? Gut 2007;56:1086-90.

[31] Bae SY, Lee KT, Lee JH, Lee JK, Lee KH, Rhee JC. Proper management and follow-up strategy of branch duct intraductal papillary mucinous neoplasms of the pancreas. Dig Liver Dis 2011 Oct 24 [Epub ahead of print].

[32] Garcea G, Dennison AR. Branch-type intraductal papillary mucinous neoplasms: an update. Pancreatology 2011;11:336-42. 